\title{
Term vs. preterm cord blood cells for the prevention of preterm brain injury
}

Jingang Li ${ }^{1}$, Tamara Yawno ${ }^{1}$, Amy Sutherland ${ }^{1}$, Jan Loose ${ }^{1}$, llias Nitsos ${ }^{1}$, Beth J. Allison ${ }^{1}$, Robert Bischof ${ }^{1}$, Courtney A. McDonald ${ }^{1}$, Graham Jenkin ${ }^{1}$ and Suzanne L. Miller ${ }^{1}$

BACKGROUND: White matter brain injury in preterm infants can induce neurodevelopmental deficits. Umbilical cord blood (UCB) cells demonstrate neuroprotective properties, but it is unknown whether cells obtained from preterm cord blood (PCB) vs. term cord blood (TCB) have similar efficacy. This study compared the ability of TCB vs. PCB cells to reduce white matter injury in preterm fetal sheep.

METHODS: Hypoxia-ischemia (HI) was induced in fetal sheep (0.7 gestation) by $25 \mathrm{~min}$ umbilical cord occlusion. Allogeneic UCB cells from term or preterm sheep, or saline, were administered to the fetus at $12 \mathrm{~h}$ after $\mathrm{HI}$. The fetal brain was collected at 10-day post $\mathrm{HI}$ for assessment of white matter neuropathology.

RESULTS: $\mathrm{HI}(n=7)$ induced cell death and microglial activation and reduced total oligodendrocytes and CNPase +myelin protein in the periventricular white matter and internal capsule when compared with control $(n=10)$. Administration of TCB or PCB cells normalized white matter density and reduced cell death and microgliosis $(P<0.05)$. PCB prevented upregulation of plasma tumor necrosis factor (TNF)-a, whereas TCB increased anti-inflammatory interleukin $(\mathrm{IL})-10 \quad(P<0.05)$. TCB, but not $\mathrm{PCB}$, reduced circulating oxidative stress.

CONCLUSIONS: TCB and PCB cells reduced preterm HIinduced white matter injury, primarily via anti-inflammatory actions. The secondary mechanisms of neuroprotection appear different following TCB vs. PCB administration.

V ery preterm birth, before 32 weeks of gestation, has significant adverse outcomes for the developing brain, leading to lifelong neurodevelopmental deficits (1). White matter injury (WMI) is the predominant form of brain injury present in about half of infants born very preterm; $25-50 \%$ of these infants will develop cognitive or behavioral deficits and up to $15 \%$ will develop cerebral palsy (1). Currently, there are no therapies for reducing WMI in preterm infants.

Preclinical animal studies demonstrate significant neuroprotective benefits of human umbilical cord blood (UCB) cells in rodent models of term HI brain injury (2-6), and subsequent clinical trials are now underway for term infant or childhood neuropathologies (6). Recent studies also show that UCB treatment is effective in HI-induced preterm brain injury $(7,8)$. In ovine studies, we have also shown that early administration of autologous or allogeneic UCB cells ameliorate gray and white matter brain damage in term and preterm sheep $(9,10)$. However, in all studies to date that have examined the neuroprotective effects of UCB, the cells were obtained from term pregnancies (2-10). This raises the important question-do UCB cells from preterm birth also demonstrate protective benefits, particularly for the preterm brain? This is critical, given that infants born preterm demonstrate the highest incidence of cerebral palsy (11), and could benefit from stem-cell therapy after birth. A recent meta-analysis demonstrates that stem-cell therapies show excellent promise for treating cerebral palsy (12), but it is also likely that treating cerebral palsy as soon as possible after birth will hold the greatest neurotherapeutic benefit $(9,13)$.

It is known that the neuroprotective actions of UCB are mediated by cell composition within the mononuclear cell (MNC) fraction. However, the cellular composition of term and preterm human UCB is quite different (14-19). The MNC fraction of UCB contains three major types of stem/progenitor cells: hematopoietic, endothelial, and mesenchymal stem/progenitor cells (HSCs, EPCs, and MSCs, respectively), along with lymphocytes and monocytes (6). It is unclear how each of these cell types contributes to neuroprotection; however, it is certain that the relative proportion and developmental profile of these stem/progenitor cells change within the fetal circulation as pregnancy progresses (14-20). We hypothesized that the neuroprotective effect of preterm cord blood (PCB) differs from term cord blood (TCB), given that cellular composition in UCB varies with gestation. Therefore, the present study compared the efficacy of allogeneic PCB vs. TCB cells in an established fetal sheep model of preterm WMI induced by acute severe hypoxia-ischemia (HI).

\footnotetext{
'The Ritchie Centre, Hudson Institute of Medical Research and Department of Obstetrics and Gynaecology, Monash University, Clayton, Victoria, Australia. Correspondence: Suzanne L. Miller (Suzie.Miller@monash.edu)

Received 12 January 2017; accepted 15 June 2017; advance online publication 16 August 2017. doi:10.1038/pr.2017.170
} 


\section{METHODS}

\section{Animals and Surgery}

The animal studies were approved by the Monash University Animal Ethics Committee (MMCA/2013/17). Surgery was performed on pregnant Merino-Border Leicester ewes at $97.5 \pm 0.2$-day gestation (term $=147$ days). Under general anesthesia, the fetus was exteriorized for insertion of fetal polyvinyl catheters $(0.8 \mathrm{~mm}$ inner diameter and $1.2 \mathrm{~mm}$ outer diameter, Dural Plastics, Australia) into a fetal femoral artery and vein. An inflatable balloon occluder (16HD, In Vivo Medical) was placed around the umbilical cord, and an amniotic catheter was inserted. Catheters were exteriorized through the maternal flank. A maternal jugular vein catheter was implanted for antibiotic administration (500 $\mathrm{mg}$ engemycin, $1 \mathrm{~g}$ ampicillin) on the day of surgery and for 3 days after. Fetal catheters were maintained by an infusion of heparinized saline and, $\sim 5$ days after surgery, recording of fetal heart rate and mean arterial pressure was commenced (Power Lab, ADInstruments, Castle Hill, Australia).

\section{Experimental Protocol}

At $102.3 \pm 0.2$-day gestation, the animals were randomized into one of the following four groups: (i) control (sham-occlusion+saline, $n=10$ ); (ii) HI (HI+saline, $n=7$ ); (iii) HI+TCB (HI+50 million TCB cells at $12 \mathrm{~h}, n=6$ ); and (iv) $\mathrm{HI}+\mathrm{PCB}(\mathrm{HI}+50$ million PCB cells at $12 \mathrm{~h}, n=6$ ). All groups comprised single fetuses, with the exception of the control and HI groups (with twins); there was no difference in sex distribution (Table 1). In our previous study (9), we observed no differences between sham-control animals with and without term UCB treatment (control+saline vs. control+TCB), and, therefore, we did not include additional control+TCB or control+PCB fetuses in this study. HI was induced by complete umbilical cord occlusion, in which the balloon occluder was filled with $2.0-2.5 \mathrm{ml}$ sterile water for 23-25 min. The occlusion was discontinued after $25 \mathrm{~min}$, or sooner if the occlusion was $>23$ min duration and the mean arterial pressure was decreased to $<8 \mathrm{~mm} \mathrm{Hg}$. Fetal arterial blood samples were collected $24 \mathrm{~h}$ before, during, and $6,12,24,48,72,120$, and $240 \mathrm{~h}$ after HI for determining blood gas parameters (ABL 800, Radiometer, Copenhagen, Denmark). Malondialdehyde (MDA) and cytokine concentrations were also assessed in selected plasma samples, stored at $-80{ }^{\circ} \mathrm{C}$ until assays were performed.

\section{Collection, Processing, and Transplantation of UCB}

Ovine UCB was collected into heparinized syringes from cesareansection delivery of term (141 days) or preterm lambs (112 days), which were separate cohorts to the animals used in this study. The blood was centrifuged at 3,100 r.p.m. for $12 \mathrm{~min}$ at room temperature, without brake, and the buffy coat layer was collected with the excess red blood cells removed using red blood cell lysis

Table 1. Fetal characteristics

\begin{tabular}{|c|c|c|c|c|}
\hline Variables & $\begin{array}{l}\text { Control, } \\
(n=10)\end{array}$ & $\begin{array}{c}\mathrm{HI}, \\
(n=7)\end{array}$ & $\begin{array}{c}\mathrm{HI}+\mathrm{TCB} \\
(n=6)\end{array}$ & $\begin{array}{c}\mathrm{HI}+\mathrm{PCB}, \\
(n=6)\end{array}$ \\
\hline Female, $n(\%)$ & $7(70)$ & $3(43)$ & $3(50)$ & $3(50)$ \\
\hline Twin, $n$ (\%) & $6(60)$ & $2(29)$ & $0(0)^{*}$ & $0(0)^{*}$ \\
\hline $\begin{array}{l}\text { UCO duration, } \\
\text { min }\end{array}$ & 0 & $24.4 \pm 0.3^{* *}$ & $24.2 \pm 0.4^{* *}$ & $24.3 \pm 0.3^{* *}$ \\
\hline Brain weight, $g$ & $34.5 \pm 0.7$ & $30.5 \pm 0.9^{* *}$ & $29.8 \pm 1.0^{* *}$ & $28.2 \pm 0.9^{* *}$ \\
\hline Body weight, kg & $2.0 \pm 0.1$ & $2.4 \pm 0.1$ & $2.1 \pm 0.2$ & $2.1 \pm 0.2$ \\
\hline $\begin{array}{l}\text { Brain/body } \\
\text { weight, g/kg }\end{array}$ & $17.6 \pm 0.7$ & $13.6 \pm 0.9^{* *}$ & $14.4 \pm 0.9^{* *}$ & $14.0 \pm 0.9^{* *}$ \\
\hline \multicolumn{5}{|c|}{$\begin{array}{l}\text { HI, hypoxia-ischemia; PCB, preterm cord blood; TCB, term cord blood; UCO, umbili- } \\
\text { cal cord occlusion. } \\
\text { Mean (\%) or mean } \pm \text { SE are presented for each group. One-way ANOVA and post } \\
\text { hoc Bonferroni tests were carried out on comparison between groups. }{ }^{*} P<0.05 \text { vs. } \\
\text { control. }{ }^{*} P<0.01 \text { vs. control. }\end{array}$} \\
\hline
\end{tabular}

buffer (Sigma-Aldrich, Melbourne, Victoria, Australia). The cells were re-suspended in fetal bovine serum with $10 \%$ dimethyl sulfoxide (Merck, Darmstadt, Germany), and cryopreserved in liquid nitrogen. TCB or PCB cells were thawed and labeled with carboxyfluorescein succinimidyl ester to facilitate cell tracking within the brain (9). Fifty million viable cells were re-suspended in $2.5 \mathrm{ml}$ sterile saline and administered to the fetus (over $3 \mathrm{~min}$ ) via the femoral vein $12 \mathrm{~h}$ after HI. On average, one dose of cells ( $\sim 50$ million) from each preterm UCB collection and one to two doses from each term UCB collection ( $50-100$ million cells) could be obtained. When cell number obtained was insufficient for 50 million total cells, cells were supplemented from another collection.

\section{Tissue Collection and Processing}

At 10 days after HI, the ewe and the fetus were killed with sodium pentobarbital IV. The fetal body and brain weights were recorded. The right cerebral hemisphere was cut transversely and immersionfixed in 10\% formalin for 5 days. Paraffin-embedded $10 \mu \mathrm{m}$ coronal forebrain sections at the level of the head of the caudate nucleus $(\mathrm{CN})$ were mounted on slides. Brain regions of interest were the subventricular zone (SVZ), periventricular white matter (PVWM), internal capsule (IC), and CN.

\section{Immunohistochemistry}

Immunohistochemistry on sections of the fetal brain was undertaken as we have described previously (9). Briefly, rabbit polyclonal oligodendrocyte transcription factor 2 (Olig2, 1:1,000; Millipore, Melbourne, Victoria, Australia) and mouse-anti-human 2',3'-cyclic nucleotide 3'-phosphodiesterase (CNPase, 1:200; Sigma Chemical, Melbourne, Victoria, Australia) antibodies were used to identify oligodendroglial lineage cells and myelinated axons, respectively. Proliferating cells were visualized with rabbit anti-human Ki-67 antibody (1:100; Dako, Santa Clara, CA, USA). Activated microglia were identified using rabbit anti-ionized calcium-binding adaptor molecule 1 (Iba-1, 1:500; Wako, Osaka, Japan). Cell death was identified using terminal deoxynucleotidyl transferase dUTP nick end labeling (TUNEL; Promega, Melbourne, Victoria, Australia). The slides were imaged at $\times 400$ magnification under light microscopy (Olympus BX-41, Melbourne, Victoria, Australia). The number of Olig2-, Ki-67-, TUNEL-, and Iba-1-immunoreactive cells per field of view were counted. The percentage of CNPase-positive area, indicative of the combined density of immature and mature oligodendrocytes and myelin protein covering of axons, was quantified by means of Image (NIH, Bethesda, MD, USA). Immunohistochemical outcomes were assessed in two sections per animal and three fields of view per region on each non-adjacent sections, with the results averaged per animal and then across animals in each group.

Double-label immunohistochemistry was used for oligodendrocyte proliferation as previously described (9). Sections were incubated with mouse anti-Olig2 (1:1,000; Millipore) and rabbit anti-Ki-67. Immunoreactivity was visualized with Alexa Fluor 594 goat antimouse (Red, 1/1,000; Molecular Probes) and Alexa Fluor 488 goat anti-rabbit (Green, 1/1,000; Molecular Probes) and viewed with fluorescence microscopy (Olympus BX-41).

\section{MDA Assay}

Lipid peroxidation was assessed via the thiobarbituric acid reactive substances, TBARS method of measuring MDA, in plasma at 6, 12, 24, 48, and $72 \mathrm{~h}$ ((ref. 9)), following the manufacturer's protocol (Cayman Chemical, Ann Arbor, MI).

\section{Cytokine Assay}

Plasma pro-inflammatory tumor necrosis factor (TNF)-a and antiinflammatory interleukin (IL)-10 concentrations were analyzed against recombinant cytokines on day 1 and day 10 after HI using standard capture enzyme-linked immunosorbent assay for ovinespecific monoclonal antibodies, as previously described (9). 


\section{Data Analysis}

Animals that completed the whole experimental course were included in data analyses. All assessments were conducted on coded slides or samples, with the examiner blinded to the group. Data are presented as mean \pm SE. Statistical analysis was performed with JMP (version 11, SAS, Cary, NC). Differences between three or more groups were analyzed by a two-way ANOVA (histology data) or one-way ANOVA (fetal weight, arterial blood gas, physiology, plasma cytokine, and MDA levels), followed by the Bonferroni post hoc test when a significant difference was found. Differences between the two groups were analyzed with either the Wilcoxon or Fisher's test. Regression analysis was performed for estimating the relationships among variables. $P<0.05$ was considered statistically significant.

\section{RESULTS}

We have previously reported data for animals that received TCB from this cohort (9). The overall fetal survival rate for the study was $85 \%$, with no significant differences in mortality between experimental groups. Fetal body weight was not different between groups. Brain weight and brain/body weight ratios were reduced in all $\mathrm{HI}$ groups $(\mathrm{HI}, \mathrm{HI}+\mathrm{TCB}$, and $\mathrm{HI}$ $+\mathrm{PCB})$, when compared with that in control $(P<0.01$, Table 1).

\section{Physiological Measures}

HI caused severe hypotension, bradycardia, acidosis, and hypoxia when compared with the controls (Figure 1), $P<0.01$. There was no difference in the duration of the $\mathrm{HI}$ insult, or any physiological parameter, measured immediately after HI, between the three $\mathrm{HI}$ groups. Following cell administration, the mean arterial pressure in $\mathrm{HI}+\mathrm{TCB}$ and $\mathrm{HI}+\mathrm{PCB}$ fetuses was increased for $24 \mathrm{~h}$; however, this was significant only in $\mathrm{HI}+\mathrm{TCB}$ fetuses $(P=0.04)$. No intergroup differences were observed.

\section{Brain Histopathology}

Ten days following fluorescent UCB administration, the cells were found within some, but not all brains (3/7 HI+TCB, 2/6 $\mathrm{HI}+\mathrm{PCB}$, Figure 2a). Where present, the cells were observed across white and gray matter, but more commonly in white matter (65\% of cells observed).

\section{White Matter Injury}

Following HI, the density of Olig2+ oligodendrocytes was reduced by 24,30 , and $41 \%$ within the SVZ, PVWM, and IC regions when compared with the density in the control fetuses $(P=0.7, P=0.1$, and $P=0.06$, respectively). Compared with HI animals, oligodendrocyte density was significantly improved toward control levels within the IC $(P=0.02)$ in $\mathrm{HI}+\mathrm{TCB}$ fetuses, and in the PVWM $(P=0.02)$ in $\mathrm{HI}+\mathrm{PCB}$ fetuses (Figure 3B). HI caused a reduction in CNPase+ oligodendrocytes and myelin density, decreased $22 \%$ in PVWM and $27 \%$ in IC $(P=0.01)$, when compared with that in control. Both TCB and PCB administration prevented the reduction of CNPase+ protein density (Figure 3B).

\section{Microglial Activation}

HI induced a significant increase in microglial cells within the PVWM and IC $(P<0.05)$ regions, when compared with those regions in control (Figure 4 ). In HI brains, the microglia demonstrated an activated morphology, with an enlarged ameboid cell body and loss of processes (Figure $4 \mathbf{b}$ ). In contrast, microglial cell density was similar in control, HI $+\mathrm{TCB}$, and HI+PCB brains, and showed characteristic morphology of ramified resting microglia, with small cell bodies and long branching processes (Figure $4 \mathbf{a}, \mathbf{c}, \mathbf{d}$ ). While both TCB and PCB reduced microglial density, TCB administration produced a more pronounced antiinflammatory effect when compared with PCB administration, with microglial cell counts in line with control values in TCB-treated animals (Figure 4) and significant reduction in more regions when comparing $\mathrm{HI}$ and $\mathrm{HI}+\mathrm{TCB}$ (PVWM: $P=0.04$; IC: $P<0.01)$ groups vs. with $\mathrm{HI}$ and $\mathrm{HI}+\mathrm{PCB}$ (PVWM: NS; IC: $P=0.03$ ) groups. Regression analysis revealed a strong negative correlation between the number of activated microglia and oligodendrocytes in PVWM $\left(R^{2}=0.62, P<0.001\right)$ and IC $\left(R^{2}=0.68, P<0.001\right)$.

\section{Cell Death and Cell Proliferation}

HI induced TUNEL+ cell death within the IC $(P<0.01$, Figure $5 \mathbf{A}, \mathbf{a}, \mathbf{b})$, and this was prevented with TCB or PCB administration (Figure 5A,a,c,d). Regression analyses showed no significant correlation between Olig2+ and TUNEL+ cell densities in any regions examined.

Cell proliferation was increased within the SVZ in HI fetuses when compared with that in controls $(P=0.03$; Figure 5B,f). Double-label immunohistochemistry demonstrated colocalization of Ki-67 and Olig2, indicative of oligodendrocyte proliferation within the SVZ following acute HI (Figure $2 \mathbf{b}$ ). There was no difference in proliferating cell number between control and HI+TCB and HI+PCB fetuses (Figure 5B,e,g,h). PCB administration reduced aberrant proliferation to a greater extent than TCB in the SVZ region (HI+PCB vs. HI; $P<0.01)$.

\section{Fetal Plasma Oxidative Stress and Cytokine Analysis}

Fetal plasma MDA, an established marker of oxidative stress, was increased at $6-12 \mathrm{~h}$ in $\mathrm{HI}$ animals and remained above baseline for $48 \mathrm{~h}$ (Table 2a). The administration of PCB did not alter the MDA profile in response to HI. In contrast, TCB treatment at $12 \mathrm{~h}$ post $\mathrm{HI}$ demonstrated an antioxidant benefit, reducing plasma MDA levels from their $12 \mathrm{~h}$ peak such that MDA was not different to basal levels by $24 \mathrm{~h}$; MDA was reduced in $\mathrm{HI}+\mathrm{TCB}$ when compared with the MDA in $\mathrm{HI}$ at $48 \mathrm{~h}(P=0.03)$. HI induced an inflammatory response shown by an increase in plasma TNF-a, as well as an endogenous anti-inflammatory response, revealed by an increase in plasma IL-10 observed at $24 \mathrm{~h}$, but not sustained to 10 days (Table $2 \mathbf{b}, \mathbf{c}$, respectively). PCB administration moderated the TNF-a response at $24 \mathrm{~h}$ and resulted in decreased plasma TNF-a in HI+PCB when compared with the plasma TNF-a in HI+TCB $(P=0.04$, Table $2 \mathbf{b})$. In contrast, 


\section{Term vs. preterm cord blood therapy Articles}
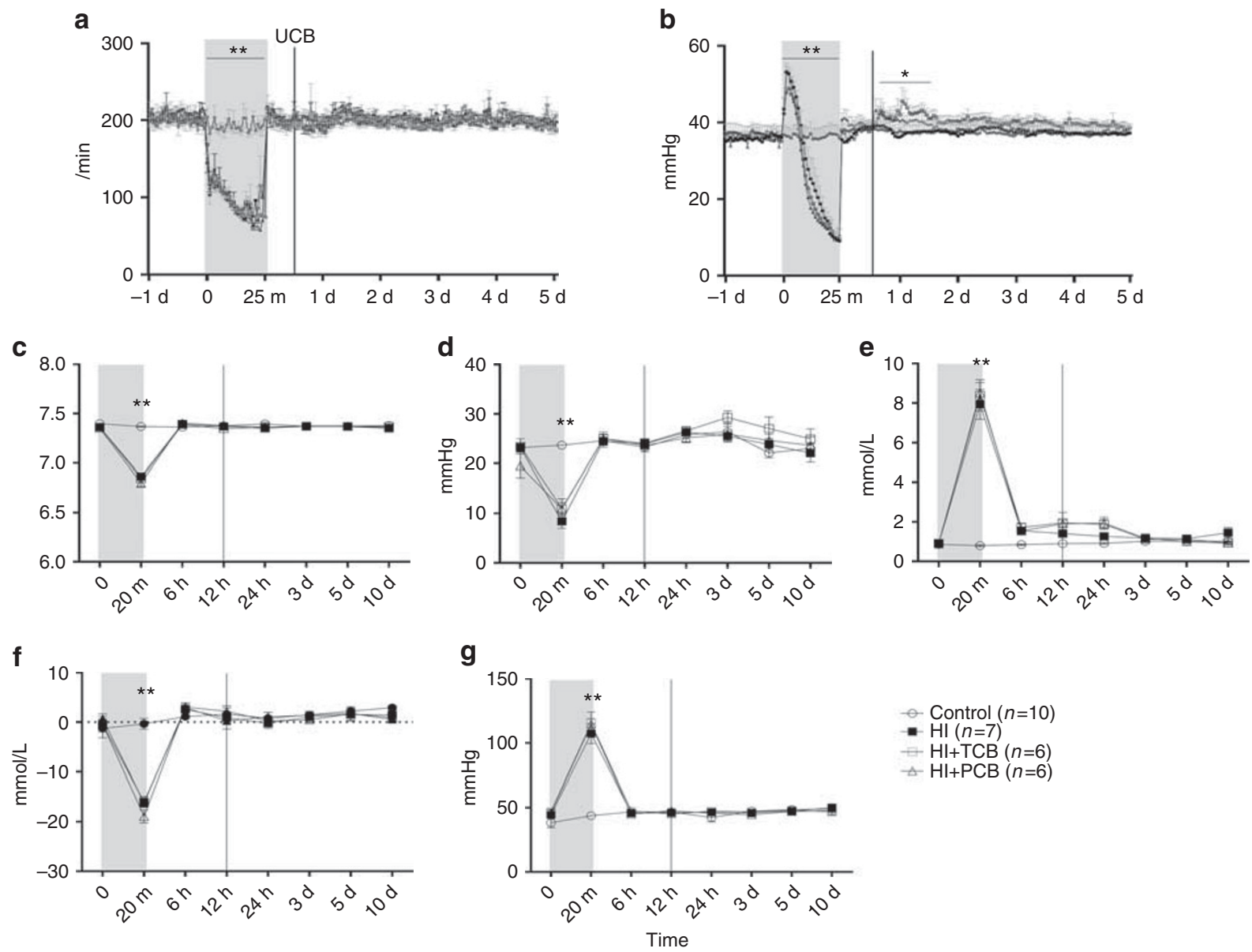

Figure 1. Physiological measurements during $\mathrm{HI}$. HI, induced by umbilical cord occlusion, is shown by the gray columns and UCB cell administration is shown by vertical lines. $\mathrm{HI}$ resulted in severe hypotension (a) and bradycardia (b), markedly reduced fetal arterial $\mathrm{pH}(\mathbf{c}), \mathrm{PaO}_{2}(\mathbf{d})$, and base excess (f), and increased $\mathrm{PaCO}_{2}(\mathbf{g})$ and lactate $(\mathbf{e})$ levels when compared with that in control groups $(P<0.01)$. Data are presented as mean $\pm \mathrm{SEM}$. BE, base excess; FHR, fetal heart rate; $\mathrm{HI}$, hypoxia-ischemia; MAP, mean arterial pressure; PCB, preterm cord blood; TCB, term cord blood; UCB, umbilical cord blood. ${ }^{*} P<0.05$ vs. control, ${ }^{* * P}<0.01$ within group comparisons vs. baseline.

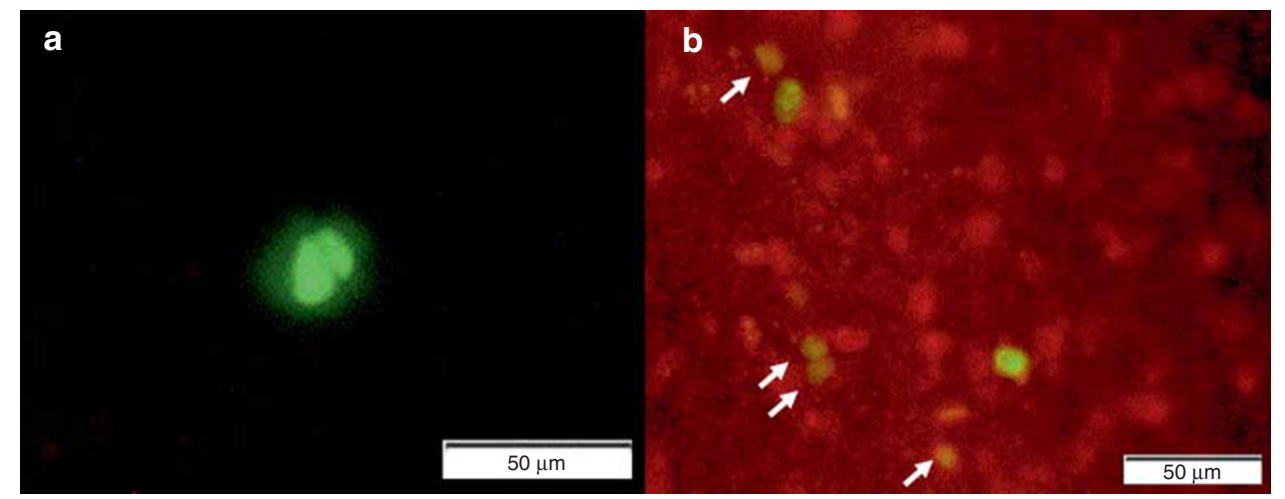

Figure 2. Fluorescent microscopy. (a) A representative microphotograph of carboxyfluorescein succinimidyl ester-labeled cells detected in the periventricular white matter of the preterm brain. (b) Double-label fluorescent immunostaining of Olig2 (red) and Ki-67 (green) with merged images in the SVZ of the brain in an HI fetus. White arrow denotes an example of a cell with dual signal. Bar $=50 \mu$ m. HI, hypoxia-ischemia; SVZ, subventricular zone.

TCB administration upregulated IL-10 at 10 days with elevated plasma IL-10 concentration in HI+TCB fetuses when compared with $\mathrm{HI}$ fetuses $(P=0.04)$; no difference was found between $\mathrm{HI}+\mathrm{PCB}$ and $\mathrm{HI}$ fetuses (Table 2c).

\section{DISCUSSION}

White matter brain injury in infants born preterm is the principal neuropathology underlying neurodevelopmental deficits including cerebral palsy (1). This study is the first to 


\section{Articles | Li et al.}

A

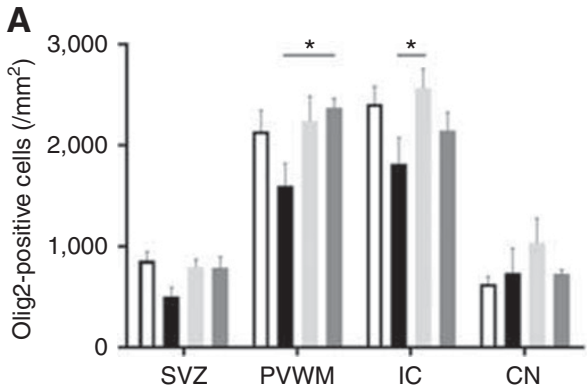

B

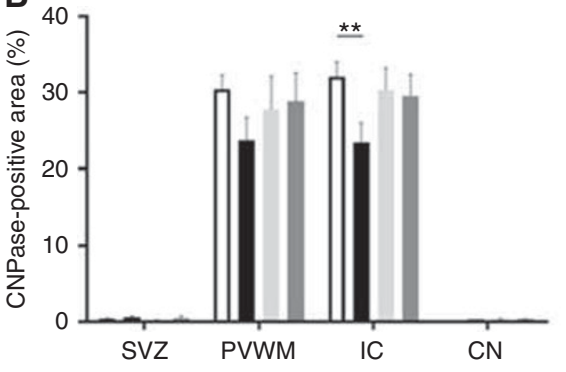

$\square$ Control $(n=10)$

$\mathrm{HI}(n=7)$

$\mathrm{HI}+\mathrm{TCB}(n=6)$

- $\mathrm{HI}+\mathrm{PCB}(n=6)$

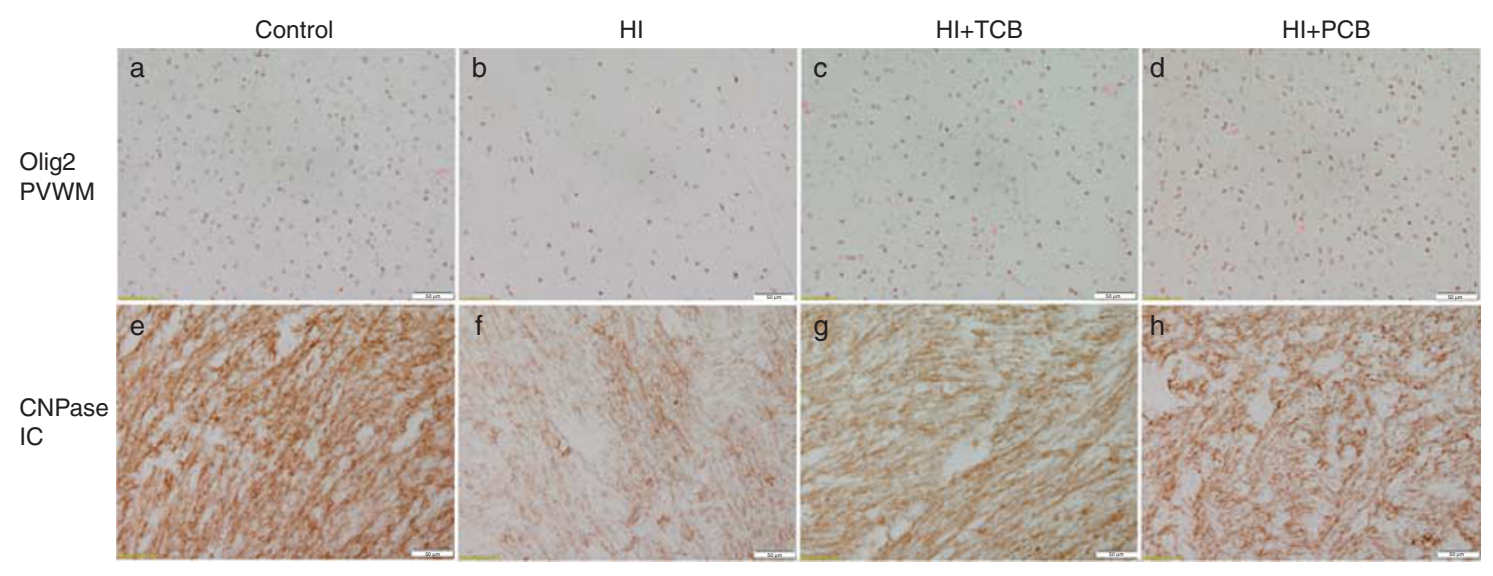

Figure 3. Effects of TCB and PCB cell on white matter cells following $\mathrm{HI}$. The graphs of the number of Olig2+ oligodendrocyte (A) and density of oligodendrocyte and myelinating axons (CNPase+, B), and the representative photomicrograph images in control (a, e), HI (b, f), HI+TCB (c, g), and HI+PCB (d, h) groups. SVZ, PVWM, IC, and CN. Data are mean \pm SEM and were analyzed using ANOVA followed by the Bonferroni post hoc test for individual brain regions. ${ }^{*} P<0.05$, ${ }^{*} P<0.01$. Bar $=50 \mu \mathrm{m}$. $\mathrm{CN}$, caudate nucleus; $\mathrm{HI}$, hypoxia-ischemia; IC, internal capsule; $P C B$, preterm cord blood; PVWM, periventricular white matter; SVZ, subventricular zone; TCB, term cord blood.

compare the neuroprotective effects of allogeneic preterm with TCB cells for preterm WMI. We show that administration of either PCB or TCB significantly reduced oligodendrocyte and CNPase+ myelin loss in response to HI, and to a similar extent. Interestingly, while both $\mathrm{PCB}$ and $\mathrm{TCB}$ preserved white matter structure, $\mathrm{PCB}$ and TCB may act differently. Both TCB and PCB mediated the neuroinflammatory response to $\mathrm{HI}$; however, TCB also demonstrated a strong antioxidant ability, whereas PCB reduced cell proliferation within the brain and reduced acute systemic inflammation. These results support early administration of allogeneic PCB or TCB to reduce WMI, and further show that the systemic and cerebral anti-inflammatory actions of cord blood therapy is central to their neuroprotective benefit.

$\mathrm{HI}$ induced by umbilical cord occlusion for $25 \mathrm{~min}$ caused WMI at 10 days post insult, with oligodendrocyte and myelin reduction across the PVWM and IC, as evidenced as a decrease in Olig2+ and CNPase+ proteins. It is well described that neuroinflammation, particularly via microglial activation, is a principal cause of preterm WMI $(20,21)$. Supporting this, we show a strong correlation between increasing activated microglia density and oligodendrocyte loss, as observed for preterm WMI induced by fetal inflammation (9). Both PCB and TCB demonstrated an excellent ability to reduce neuroinflammation, reducing the number of activated microglia and maintaining microglial phenotype in a resting state. HI also increased cell proliferation within the SVZ, a response recognized as an aberrant production of oligodendrocytes with limited functionality (22). Both PCB and TCB significantly reduced total oligodendrocyte cell death and restored CNPase+ oligodendrocyte and myelin density. However, PCB treatment normalized SVZ cell proliferation, whereas TCB did not, indicating that PCB may be more effective at restoring the balance between cell death and proliferation.

In this study, TCB cell administration reduced circulating markers of oxidative stress following HI. In vitro evidence has shown that stem cells possess antioxidant capacity $(23,24)$, with more immature and younger cells demonstrating a stronger antioxidant ability (25). The current study, the first in vivo assessment of $\mathrm{PCB}$ vs. ТCB, suggests that ТСB has a greater antioxidant capacity than PCB. However, we did observe a large variability and higher baseline values in MDA concentration within HI+PCB fetuses (Table 2a), which might be due to the severity of the initial injury and somewhat limits interpretation of these data. Similarly, TCB, but not $\mathrm{PCB}$, induced an increase in systemic anti-inflammatory IL-10 concentration following HI (Table 2c). IL-10 is primarily produced by monocytes, and to a lesser extent by lymphocytes (26), and an increased proportion of monocytes 

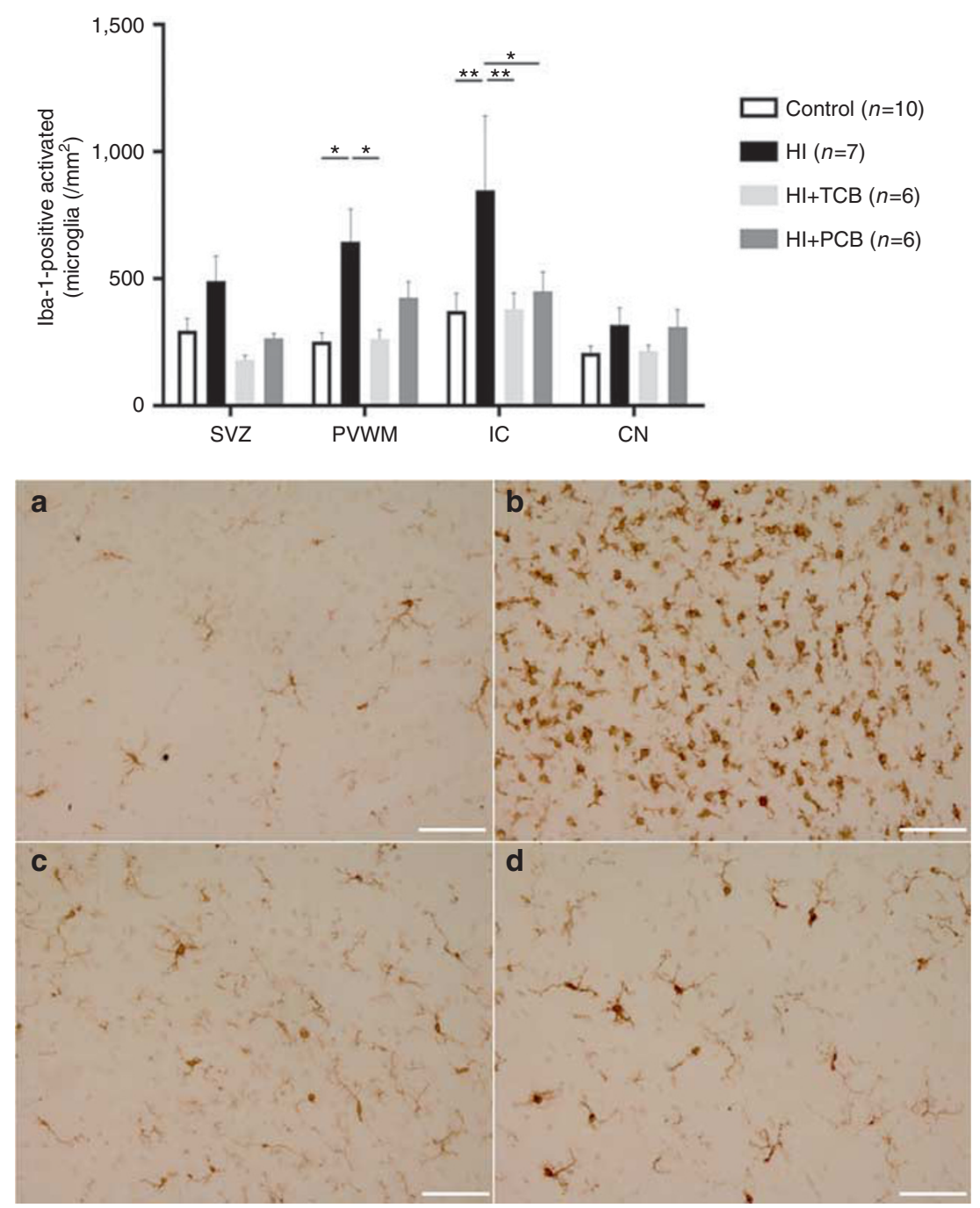

Figure 4. Effects of TCB and PCB cell following $\mathrm{HI}$ on the density of Iba-1+ activated microglia. The photomicrograph images show that lba-1+ inflammatory cells in control brains exhibited the characteristics of ramified (resting) microglia, with small cell bodies and long branching processes (a). However, the cells in $\mathrm{Hl}$ group show loss of microglial branching and transformation into rounded macrophages (b). The changes are to a large extent reversed following TCB and PCB cell administration at $12 \mathrm{~h}$ (c,d). SVZ, PVWM, IC, and CN. Data are mean \pm SEM, and were analyzed using ANOVA followed by the Bonferroni post hoc test for individual brain regions. ${ }^{*} P<0.05,{ }^{*} P<0.01$. Bar $=50 \mu \mathrm{m}$. CN, caudate nucleus; HI, hypoxia-ischemia; IC, internal capsule; PCB, preterm cord blood; PVWM, periventricular white matter; SVZ, subventricular zone; TCB, term cord blood.

in TCB might account for this result (27). In contrast, only PCB moderated the pro-inflammatory TNF-a response following HI (Table 2c). Suppressing pro-inflammatory cytokines (e.g., TNF-a and IL-1) is one of the modes of action of MSCs (28), and increased MSCs in PCB are likely to be a principal reason for this systemic anti-inflammatory effect (15). Although the cytokine data show wide variability, results indicate that the neuroprotective actions demonstrated by PCB and TCB may be mediated, at least in part, by different secondary mechanisms.

We observed an acute response of blood pressure elevation by allogeneic UCB cell administration, albeit this was statistically significant in the TCB-treated group only. In our previous study, we examined the neuroprotective effects of autologous UCB cells in term HIE lambs and did not observe any cardiovascular effects of the cells (10). That allogeneic UCB cell therapy causes a systemic response is not entirely surprising, considering that the administration of human (allogeneic) UCB to children with cerebral palsy induced an immune/inflammatory reaction, and children who showed the greatest response also demonstrated the most improved functional outcomes (29). We utilized allogeneic rather than autologous UCB administration for the following two reasons: (i) to avoid possible harm to preterm fetuses by withdrawing a large volume of UCB in this in utero model and (ii) to compare PCB with TCB in the same immunological (allogeneic) setting. Our findings also have implications for the use of autologous preterm UCB for neuroprotection. Unlike hypoxic-ischemic encephalopathy at term, the timely diagnosis of preterm WMI is extremely challenging (6). In 


\section{Articles | Li et al.}
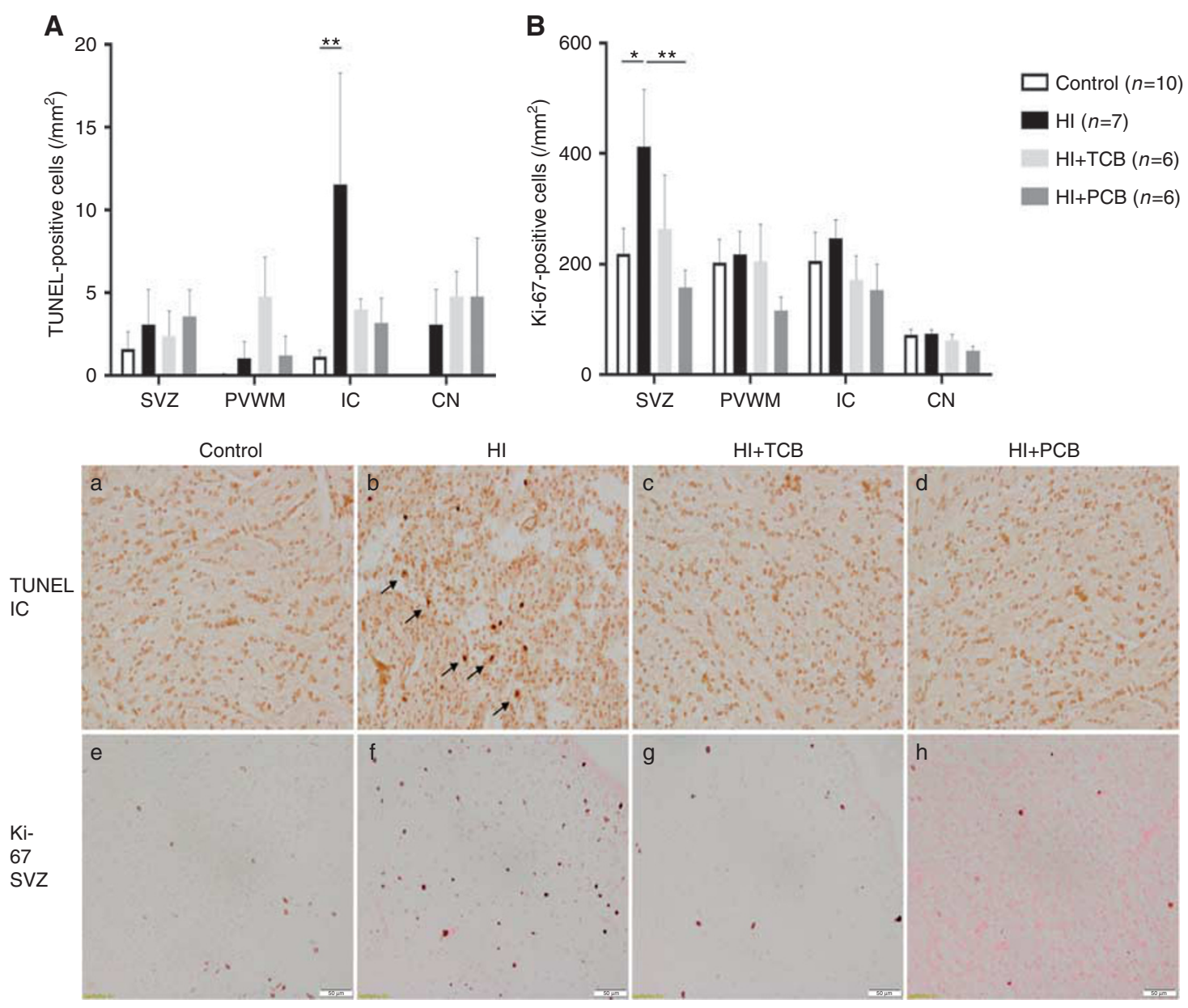

Figure 5. Effects of TCB cell and PCB cell on cell death and cell proliferation following HI. The graphs of the density of TUNEL+ cell death (A) and Ki-67+ proliferating cells (B), and the representative photomicrograph images in control (a,e), HI (b,f), HI+TCB (c,g), and HI+PCB (d,h) groups. Black arrows show examples of TUNEL-positive cells (b). Data are mean \pm SEM, and were analyzed using ANOVA followed by the Bonferroni post hoc test for individual brain regions. ${ }^{*} P<0.05,{ }^{* *} P<0.01$. Bar $=50 \mu \mathrm{m}$. $\mathrm{HI}$, hypoxia-ischemia; $\mathrm{PCB}$, preterm cord blood; TCB, term cord blood; TUNEL, terminal deoxynucleotidyl transferase dUTP nick end labeling.

Table 2a. MDA $(\mu \mathrm{M})$

\begin{tabular}{lcccc}
\hline & Control & $\mathrm{HI}$ & $\mathrm{HI}+\mathrm{TCB}$ & $\mathrm{HI}+\mathrm{PCB}$ \\
\hline Base & $9.7 \pm 2.3$ & $9.6 \pm 2.3$ & $8.0 \pm 0.9$ & $12.9 \pm 3.4$ \\
$6 \mathrm{~h}$ & $6.5 \pm 2.0$ & $11.2 \pm 2.0^{\mathrm{a}}$ & $12.5 \pm 1.4^{\mathrm{a}}$ & $18.2 \pm 9.6$ \\
$12 \mathrm{~h}$ & $9.0 \pm 2.5$ & $17.8 \pm 2.5^{\mathrm{a}}$ & $15.5 \pm 3.1$ & $16.6 \pm 7.5$ \\
$24 \mathrm{~h}$ & $6.7 \pm 1.7$ & $17.1 \pm 1.7^{\mathrm{a}}$ & $10.6 \pm 2.1$ & $20.6 \pm 9.5$ \\
$48 \mathrm{~h}$ & $9.4 \pm 2.3$ & $18.1 \pm 2.3^{\mathrm{a}}$ & $10.2 \pm 2.4^{\mathrm{b}}$ & $17.1 \pm 3.0$ \\
$72 \mathrm{~h}$ & $8.3 \pm 4.7$ & $11.7 \pm 4.7$ & $9.1 \pm 4.2$ & $13.7 \pm 3.3$
\end{tabular}

$\mathrm{HI}$, hypoxia-ischemia; MDA, malondialdehyde; $\mathrm{PCB}$, preterm cord blood; $\mathrm{TCB}$, term cord blood.

${ }^{\mathrm{a} p}<<0.05$ vs. control. ${ }^{\mathrm{b}} \mathrm{P}<0.05$ vs. HI.

this study we administered cells at $12 \mathrm{~h}$ after $\mathrm{HI}$, as the therapeutic window of UCB therapy for optimal neuroprotection is up to 3 days after HI $(9,13,30)$. The collection and administration of autologous UCB soon after birth may provide increased benefit in high-risk very preterm or extremely preterm infants. In this situation, autologous cells are readily available, free from immunological side effects, and potentially have a cellular composition that is developmentally appropriate with a high relative proportion of stem/ progenitor cells. In contrast, UCB banks predominantly collect from full-term births. The volume of available UCB for collection is correlated with birth weight (i.e., $18-23 \mathrm{ml} /$ $\mathrm{kg})(31,32)$, and therefore, whereas the volume of UCB for collection is low at preterm birth, the total number of cells would be sufficient for treatment of a preterm infant $\left(62 \pm 31 \mathrm{ml}, 3.6 \times 10^{8}\right.$ cells at $25-33$ weeks vs. $102 \pm 30 \mathrm{ml}$, $11.3 \times 10^{8}$ cells at term (14)). It should, however, be considered that, although volume and cell number are feasible for PCB transplantation in preterm infants $(19,31)$, preterm birth is frequently associated with obstetric complications (e.g., chorioamnionitis and intrauterine growth restriction), which may alter the volume and cell composition of UCB $(33,34)$, and may prohibit autologous PCB administration.

It is likely that the differential effects of PCB vs. TCB are mediated via individual cell composition. HSCs (CD34+, $\mathrm{CD} 45+)$ give rise to multiple blood lineages and comprise the largest progenitor cell population in human UCB $(\sim 0.5 \%$ of 
Table 2b. TNF-a (pg/ml)

\begin{tabular}{lcccc}
\hline & Control & $\mathrm{HI}$ & $\mathrm{HI}+\mathrm{TCB}$ & $\mathrm{HI}+\mathrm{PCB}$ \\
\hline $24 \mathrm{~h}$ & $2.9 \pm 0.5$ & $4.0 \pm 1.2$ & $5.0 \pm 0.9$ & $1.6 \pm 0.7^{\mathrm{a}}$ \\
$10 \mathrm{~d}$ & $3.1 \pm 1.0$ & $2.7 \pm 2.7$ & $2.6 \pm 1.0$ & $1.3 \pm 0.8$ \\
\hline
\end{tabular}

d, day; HI, hypoxia-ischemia; PCB, preterm cord blood; TCB, term cord blood; TNF, tumor necrosis factor.

${ }^{\mathrm{a}} \mathrm{P}<0.05$ vs. HI+TCB.

Table 2c. IL-10 (BU/ml)

\begin{tabular}{lcccc}
\hline & Control & $\mathrm{Hl}$ & $\mathrm{Hl}+\mathrm{TCB}$ & $\mathrm{HI}+\mathrm{PCB}$ \\
\hline $24 \mathrm{~h}$ & $0.7 \pm 0.7$ & $7.0 \pm 3.5$ & $5.4 \pm 4.1$ & $2.6 \pm 1.4$ \\
$10 \mathrm{~d}$ & $13.5 \pm 2.0$ & $11.3 \pm 1.4$ & $16.0 \pm 1.4^{\mathrm{a}}$ & $12.0 \pm 1.4$ \\
\hline
\end{tabular}

d, day; HI, hypoxia-ischemia; IL, interleukin; PCB, preterm cord blood; TCB, term cord blood.

${ }^{\mathrm{a}} \mathrm{P}<0.05$ vs. $\mathrm{HI}$

MNCs) (14). EPCs have a potent ability for neovascularization (35), accounting for 1-2\% of the HSC-containing CD34+ cell fraction in UCB. MSCs (CD34-, CD45-, and plastic adherent) are multipotent stem cells, capable of differentiating into multiple lineages, and have immunomodulatory/antiinflammatory properties and high proliferation capacity $(28,36)$. The majority of studies investigating UCB for perinatal brain injury have administered xenogeneic (human) whole MNCs (2-6); however, UCB-CD34+ cells, UCB-MSCs, as well as monocytes and lymphocytes in UCB may all contribute to neuroprotection $(37-40)$. The relative proportion and developmental profile of these stem/progenitor cells change as pregnancy progresses (14-19), suggesting that the neuroprotective capacity of UCB may also change over gestation. The proportion of HSCs decreases over the last trimester, whereas MNCs remain constant $(14,17,19)$. MSCs account for only $0.002 \%$ of MNCs in term UCB, but increase with decreasing gestation, with a 10-fold increase at 28-31 weeks compared with term (16), and a $>40$-fold increase at 24-28 weeks' gestation (15). Furthermore, PCB has a higher proportion of immature stem/progenitor cells (14-19), more immature subsets of lymphocytes, and a decreasing monocyte population with increasing gestation (17,27). This cell composition may be critical for normal developmental processes within the preterm brain.

We acknowledge that our ability to compare the cellular composition of ovine PCB and TCB was limited because of a lack of suitable monoclonal antibodies for ovine cell surface markers. However, we are now characterizing ovine cord blood and, to date, have observed that ovine PCB contains a greater frequency of colony-forming adherent cells (MSCs and EPCs) when compared with TCB (personal observation, Jingang Li), as occurs in human $\operatorname{UCB}(15,16)$. This is indicative that across species, or at least in humans and sheep, there is a similar gestational profile for change in cord blood cell content. We used CNPase as an immunohistochemical marker of white matter integrity within the developing brain, where CNPase stains immature/mature oligodendrocytes and is a structural protein in the myelin membrane, constituting $\sim 5 \%$ of myelin protein in the adult brain $(41,42)$. The reduction in CNPase+ staining observed in this study could be attributed to a delay in oligodendrocyte maturation and/or reduced myelination. A further limitation of the current study was that relatively small group numbers meant that we were unable to examine whether male and female fetuses respond to term and preterm UCB in a qualitatively similar manner.

In summary, UCB obtained from preterm or term pregnancy was effective at reducing preterm WMI following acute HI. Both PCB and TCB reduced the neuroinflammatory response in the preterm brain-a principal mechanism of oligodendrocyte cell loss and hypomyelination. However, the secondary mechanisms of neuroprotection appear to be different in response to $\mathrm{PCB}$ and TCB administration. PCB suppressed acute systemic inflammation by reducing plasma TNF-a following HI, whereas TCB induced a late increase in anti-inflammatory cytokine IL-10 and decreased systemic oxidative stress. Changes in stem/progenitor cell composition of UCB over the course of gestation are likely to influence the mode/s of neuroprotective action.

\section{STATEMENT OF FINANCIAL SUPPORT}

This work was made possible with financial support from Inner Wheel Australia, L.E.W. Carty Charitable Fund, Victorian Government Operational Infrastructure Support Program, an NHMRC Australia Project Grant (APP1081516) and ARC Future Fellowship to SLM.

Disclosure: The authors declare no conflict of interest.

\section{REFERENCES}

1. Volpe JJ. Cerebral white matter injury of the premature infant: more common than you think. Pediatrics 2003;112:176-80.

2. Pimentel-Coelho PM, Magalhães ES, Lopes LM, et al. Human cord blood transplantation in a neonatal rat model of hypoxic-ischemic brain damage: Functional outcome related to neuroprotection in the striatum. Stem Cells Dev 2010;19:351-8.

3. Meier C, Middelanis J, Wasielewski B, et al. Spastic paresis after perinatal brain damage in rats is reduced by human cord blood mononuclear cells. Pediatr Res 2006;59:244-9.

4. Geissler M. Human umbilical cord blood cells restore brain damage induced changes in rat somatosensory cortex. PLoS ONE 2011;6:e20194.

5. Wasielewski B, Jensen A, Roth-Härer A, Dermietzel R, Meier C. Neuroglial activation and $\mathrm{Cx} 43$ expression are reduced upon transplantation of human umbilical cord blood cells after perinatal hypoxicischemic injury. Brain Res 2012;1487:39-53.

6. Li J, McDonald C, Fahey MC, Jenkin G, Miller SL. Could cord blood cell therapy reduce preterm brain injury? Front Neurol 2014;5:200.

7. Drobyshevsky A, Cotten CM, Shi Z, et al. Human umbilical cord blood cells ameliorate motor deficits in rabbits in a cerebral palsy model. Dev Neurosci 2015;37:349-62.

8. Hall AA, Guyer AG, Leonardo CC, et al. Human umbilical cord blood cells directly suppress ischemic oligodendrocyte cell death. J Neurosci Res 2009;87:333-41.

9. Li J, Yawno T, Sutherland A, et al. Preterm white matter brain injury is prevented by early administration of umbilical cord blood cells. Exp Neurol 2016;283:179-87.

10. Aridas JDS, McDonald CA, Paton MCB, et al. Cord blood mononuclear cells prevent neuronal apoptosis in response to perinatal asphyxia in the newborn lamb. J Physiol 2016;594:1421-35. 
11. Trønnes H, Wilcox AJ, Lie RT, Markestad T, Moster D. Risk of cerebral palsy in relation to pregnancy disorders and preterm birth: a national cohort study. Dev Med Child Neurol 2014;56:779-85.

12. Novak I, Walker K, Hunt R, Wallace E, Fahey M, Badawi N. Concise review: stem cell interventions for people with cerebral palsy: systematic review with meta-analysis. Stem Cells Transl Med 2016;5: 1014-25.

13. Boltze J, Schmidt UR, Reich DM, et al. Determination of the therapeutic time window for human umbilical cord blood mononuclear cell transplantation following experimental stroke in rats. Cell Transplant 2012;21:1199-211.

14. Solves P, López M, Mirabet V, Blanquer A, Roig R, Perales A. Characteristics of umbilical cord blood units collected from preterm deliveries. Gynecol Obstetr Invest 2009;68:181-5.

15. Javed MJ, Mead LE, Prater D, et al. Endothelial colony forming cells and mesenchymal stem cells are enriched at different gestational ages in human umbilical cord blood. Pediatr Res 2008;64:68-73.

16. Jain A, Mathur N, Jeevashankar M, et al. Does mesenchymal stem cell population in umbilical cord blood vary at different gestational periods? Indian J Pediatr 2013;80:375-9.

17. Cervera A, Lillo R, García-Sánchez F, Madero L, Madero R, Vicario JL. Flow cytometric assessment of hematopoietic cell subsets in cryopreserved preterm and term cord blood, influence of obstetrical parameters, and availability for transplantation. Am J Hematol 2006;81:397-410.

18. Gasparoni A, Ciardelli L, Avanzini MA, et al. Immunophenotypic changes of fetal cord blood hematopoietic progenitor cells during gestation. Pediatr Res 2000;47:825-9.

19. Surbek DV, Holzgreve W, Jansen W, et al. Quantitative immunophenotypic characterization, cryopreservation, and enrichment of second-and third-trimester human fetal cord blood hematopoietic stem cells (progenitor cells). Am J Obstetr Gynecol 1998;179:1228-33.

20. Czeh M, Gressens P, Kaindl AM. The yin and yang of microglia. Dev Neurosci 2011;33:199-209.

21. Hagberg H, Mallard C, Ferriero DM, et al. The role of inflammation in perinatal brain injury. Nat Rev Neurol 2015;11:192-208.

22. Buser JR, Maire J, Riddle A, et al. Arrested preoligodendrocyte maturation contributes to myelination failure in premature infants. Ann Neurol 2012;71:93-109.

23. Arien-Zakay H, Lecht S, Bercu MM, et al. Neuroprotection by cord blood neural progenitors involves antioxidants, neurotrophic and angiogenic factors. Exp Neurol 2009;216:83-94.

24. Valle-Prieto A, Conget PA. Human mesenchymal stem cells efficiently manage oxidative stress. Stem Cells Dev 2010;19:1885-93.

25. Ito K, Hirao A, Arai F, et al. Regulation of oxidative stress by ATM is required for self-renewal of haematopoietic stem cells. Nature 2004;431: 997-1002.

26. Said EA, Dupuy FP, Trautmann L, et al. Programmed death-1-induced interleukin-10 production by monocytes impairs CD4(+) T cell activation during HIV infection. Nat Med 2010;16:452-9.
27. Christensen RD, Del Vecchio A, Henry E. Expected erythrocyte, platelet and neutrophil values for term and preterm neonates. J Maternal Fetal Neonatal Med 2012;25:77-9.

28. Prockop DJ, Youn Oh J. Mesenchymal stem/stromal cells (MSCs): role as guardians of inflammation. Mol Ther 2012;20:14-20.

29. Kang M, Min K, Jang J, et al. Involvement of immune responses in the efficacy of cord blood cell therapy for cerebral palsy. Stem Cells Dev 2015;24:2259-68.

30. Yu G, Borlongan CV, Stahl CE, et al. Systemic delivery of umbilical cord blood cells for stroke therapy: a review. Restorative Neurol Neurosci 2009;27:41-54.

31. Surbek DV, Glanzmann R, Senn HP, Hoesli I, Holzgreve W. Can cord blood be used for autologous transfusion in preterm neonates? Eur J Pediatrics 2000;159:790-1.

32. Hosono S, Hine K, Nagano N, et al. Residual blood volume in the umbilical cord of extremely premature infants. Pediatr Int 2015;57:68-71.

33. Kotiranta-Ainamo A, Apajasalo M, Pohjavuori M, Rautonen N, Rautonen J. Mononuclear cell subpopulations in preterm and full-term neonates: independent effects of gestational age, neonatal infection, maternal pre- eclampsia, maternal betamethason therapy, and mode of delivery. Clin Exp Immunol 1999;115:309-14.

34. Jones J, Stevens CE, Rubinstein P, Robertazzi RR, Kerr A, Cabbad MF. Obstetric predictors of placental/umbilical cord blood volume for transplantation. Am J Obstetr Gynecol 2003;188:503-9.

35. Larrivée B, Niessen K, Pollet I, et al. Minimal contribution of marrowderived endothelial precursors to tumor vasculature. J Immunol 2005;175: 2890-9.

36. Lee OK, Kuo TK, Chen WM, Lee KD, Hsieh SL, Chen TH. Isolation of multipotent mesenchymal stem cells from umbilical cord blood. Blood 2004;103:1669-75.

37. Tsuji M, Taguchi A, Ohshima M, et al. Effects of intravenous administration of umbilical cord blood CD34+ cells in a mouse model of neonatal stroke. Neuroscience 2014;263:148-58.

38. Xia G, Hong X, Chen X, Lan F, Zhang G, Liao L. Intracerebral transplantation of mesenchymal stem cells derived from human umbilical cord blood alleviates hypoxic ischemic brain injury in rat neonates. J Perinatal Med 2010;38:215-21.

39. Shahaduzzaman M, Golden JE, Green S, et al. A single administration of human umbilical cord blood $\mathrm{T}$ cells produces long-lasting effects in the aging hippocampus. Age 2013;35:2071-87.

40. Womble TA, Green S, Shahaduzzaman M, et al. Monocytes are essential for the neuroprotective effect of human cord blood cells following middle cerebral artery occlusion in rat. Mol Cell Neurosci 2014;59:76-84.

41. Alves de Alencar Rocha AK, Allison BJ, Yawno T, et al. Early- versus late-onset fetal growth restriction differentially affects the development of the fetal sheep brain. Dev Neurosci 2017;39:141-155.

42. Trapp BD, Bernier L, Andrews SB, Colman DR. Cellular and subcellular distribution of 2',3'-cyclic nucleotide 3'-phosphodiesterase and its mRNA in the rat central nervous system. J Neurochem 1988;51:859-68. 